\title{
Unified comprehension of the kinetics of isothermal ceramic processes under field-free and resonant wave-field conditions
}

\author{
B. Wong ${ }^{1 *}$ \\ Consultant, Torrance, California, 90503, USA
}

\begin{abstract}
This paper proposes a unified comprehension of the isothermal ceramic process kinetics stemming from mesoscopic irreversible thermodynamics. Accordingly, a unified process kinetic equation (UPKE) is derived, which predicts that the global isothermal process rate of any ceramic process is, in general, nonlinearly related both to its activation energy and its affinity. Nevertheless, for a low-affinity ceramic process conducted either in a field-free or resonant wave-field condition, its global isothermal rate, according to the proposed UPKE, is approximately linearly related to its affinity in the spirit of Fick's diffusion law. Therefore, the rate enhancement of a low-affinity process occurring in any resonant wave-field may be caused either by a reduction in activation energy, as in microwave-enhanced sintering, or by activation energy reduction along with an affinity augmentation, as in microwaveassisted glass-crystallization. Conversely, for a high-affinity ceramic process, e.g., a chemical reaction, the 'degenerate' UPKE predicts that its kinetics is exclusively dictated by the activation energy in the spirit of Arrhenius's rate law. Hence, isothermal rate enhancements of chemical reactions in ceramic processing under resonant wave-field conditions are believed to predominantly result from a field-induced reduction in activation energy.
\end{abstract}

Keywords: irreversible thermodynamics, mesoscopic (meso), total chemical potential, unified process kinetic equation (UPKE), resonant wave-field (WF), rate (kinetics) enhancement.

\section{INTRODUCTION}

In a recent publication [1], this author presented a fieldaugmented irreversible thermodynamic approach to semiquantitatively describe materials process rate enhancements under coherent, polarized, resonant (CPR) microwave irradiation. A linear rate-force kinetics for ceramic processes under resonant microwaves was thus formulated. Yet, the rationale accordingly developed was strictly applicable to the understanding of the enhanced kinetics of microwave-assisted, low-affinity (diffusion-controlled) ceramic processes including accelerated isothermal solidstate powder-sintering and glass-crystallization under microwaves. This paper presents a refined irreversible thermodynamic approach stemming from a unique mesoscopic process-energetic model and develops a unified comprehension of all isothermal ceramic process kinetics. Accordingly, a unified process kinetic equation (UPKE) is subsequently derived for characterizing kinetics of ceramic processes of both low and high affinities in the presence or absence of a resonant wave-field.

\section{ISOTHERMAL PROCESS KINETICS - A MESOSCOPIC IRREVERSIBLETHERMODYNAMIC MODELING}

In irreversible thermodynamics, as long as a stationary, nonequilibrium, macroscopic system complies with the

*boonandcarrie@gmail.com

Dhttps://orcid.org/0000-0003-3248-0144 local instantaneous equilibrium hypothesis, the 'local' $2^{\text {nd }}$ law stated in Eq. A holds for any mesoscopic, isothermal, isobaric, irreversible matter transformation or transport process occurring within the system [2-5]:

$\mathrm{T} . \sigma(\xi)=\varrho(\xi) \cdot[-\partial \mu(\xi) / \partial \xi]>0$

where $\sigma(\xi)$ : the entropy production rate of a meso-volume cell in the system due to matter flow along any isothermal, internal process coordinate, $\xi$; T: thermodynamic temperature; $\varrho(\xi)$, $\mu(\xi)$ : local rate and total chemical potential, respectively, of the mesoscopic matter process along $\xi$; and $-\partial \mu(\xi) / \partial \xi$ : conjugate molar force driving the meso-process. According to Eq. A, $\varrho(\xi)$ and $-\partial \mu(\xi) / \partial \xi$ must have the same sign. One may thus apply Onsager's phenomenological rate law to describe any mesoscopic materials process occurring within a global nonequilibrium system as:

$\varrho(\xi)=\lambda(\xi) \cdot[-\partial \mu(\xi) / \partial \xi]$

where $\lambda(\xi)$ : a $\xi$-dependent, (Onsager's) phenomenological kinetic coefficient. The validity of Eq. B sustains for most physical and chemical processes encountered in ceramic processing; its applicability only fails in domains of extremely fast processes either with abnormally low activation energies or driven by very high-frequency/ intensity external fields $[1,2,6]$.

A thermodynamically-based model for materials process kinetics: according to mesoscopic irreversible thermodynamics [4], most isothermal solid-state processes, kinetically, obey Onsager's phenomenological rate law, i.e., Eq. B. At the same time energetically, typical matter 
transformations or transports occurring during these processes would proceed along an activated, Prigoginetype internal (mesoscopic) coordinate. Consequently, the total chemical potential, $\mu(\xi)$, along the mesoscopic process coordinate, $\xi$, of any isothermal solid-state process: converting initial-state $\alpha$ into final-state $\beta$, as demonstrated in Fig. 1, can be evaluated (see analysis in Appendix I):

$\mu(\xi)=\mu(\alpha)+R \cdot T \cdot \ln \left(\gamma \cdot c(\xi) / c_{\mathrm{o}}\right)+Æ$

where $\mu(\alpha)$ : total chemical potential of any pure solid matter at its initial state, $\alpha$; R: universal gas constant; $\gamma$ : activity coefficient; $\gamma=1$ for ideal mixings, equals a dimensionless constant for dilute mixings; $c(\xi), c_{0}$ : concentration of the transition complex created along the coordinate $\xi$ and the standard-state concentration ( $\equiv 1 \mathrm{M})$, respectively, both at the same temperature; $Æ$ : activation energy of the isothermal process. Note: $Æ>R T$ and is assumed uniform along $\xi$.

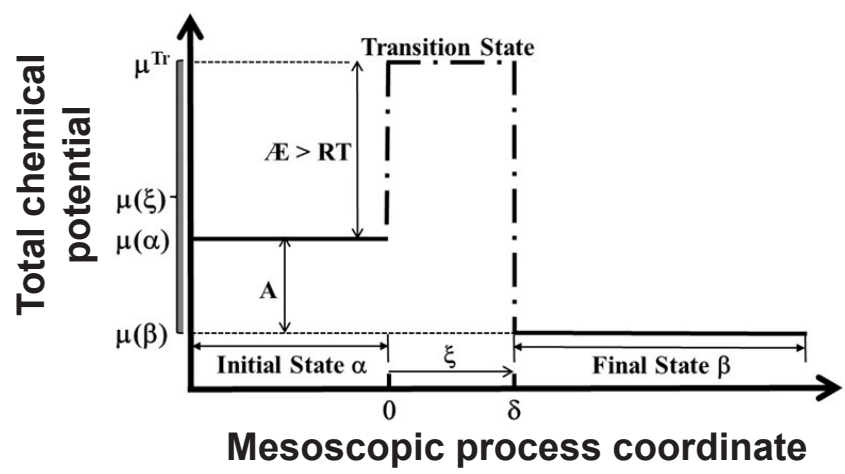

Figure 1: A schematic showing the total chemical potential variation along any isothermal mesoscopic process coordinate, $\xi$. The transformation/transport process, converting matter from its initial-state $\alpha$ into its final-state $\beta$, is envisioned as a diffusion, transferring the transition complex along $\xi$ over the activation energy barrier, $Æ$, through the entire meso-process path-length, $\delta$, and subsequently into the final-state $\beta$. Note: $Æ>R T$.

Deriving a unified process kinetic equation (UPKE): applying Eq. C, one may first express the concentration of the transition complex formed along the coordinate, $\xi$, as a function of the total chemical potential, $\mu(\xi)$, by:

$\mathrm{c}(\xi)=\left(\mathrm{c}_{\mathrm{o}} / \gamma\right) \cdot\{\exp \{[\mu(\xi)-\mu(\alpha)] /(\mathrm{R} \cdot \mathrm{T})\} \cdot \exp [-Æ \mathrm{E} /(\mathrm{R} \cdot \mathrm{T})]\}$

Next, via a direct comparison between the formalisms of matter fluxes in irreversible thermodynamics and classical mechanics, one may also relate Onsager's phenomenological coefficient, $\lambda(\xi)$, as stated in Eq. B, to the concentration of the transition complex, $c(\xi)$, by [5]:

$\lambda(\xi)=$ III.c $(\xi)$

where III is the molar mobility of the transition complex created in the process, a material constant at a given temperature under the standard-state pressure ( $\equiv 1 \mathrm{~atm})$. Now, combining Eqs. D and E, followed by substituting the subsequent result of $\lambda(\xi)$ into Eq. B, one then obtains a revised version of Onsager's phenomenological rate law, which describes the local rate of any isothermal meso-matter process as:

$\mathrm{Q}(\xi)=($ III.c. $/ \gamma / \gamma) \cdot \exp [-Æ /(R \cdot T)]$

$\exp \{[\mu(\xi)-\mu(\alpha)] /(\mathrm{R} . \mathrm{T})\} \cdot[-\partial \mu(\xi) / \partial \xi]=$

(R.T.III.c $/ \gamma) \cdot \exp [-Æ /(R . T)]$.

$\{-\partial / \partial \xi\{\exp \{[\mu(\xi)-\mu(\alpha)] /($ R.T $)\}\}\}$

Eq. F suggests that the local rate of any isothermal mesomatter process is neither linearly related to its activation energy, Æ, nor to its driving force, $-\partial \mu(\xi) / \partial \xi$. Since the local rate of any stationary activated process could be approximated to be constant everywhere along its meso-path [4], integrating Eq. F along the meso-coordinate, $0 \leq \xi \leq \delta$ (Fig. 1), using the initial state: $\mu(\xi=0) \equiv \mu(\alpha)$, and the final state: $\mu(\xi=\delta) \equiv \mu(\beta)$, one may then express the global steadystate rate, $\varrho$, for any isothermal matter process, $\alpha \rightarrow \beta$, as:

$$
\begin{aligned}
& \mathrm{Q}=\mathrm{R} \cdot \mathrm{T} \text {.III.c } \mathrm{c}_{\mathrm{o}} /(\gamma \cdot \delta) \cdot \exp [-Æ /(\mathrm{R} \cdot \mathrm{T})] . \\
& \{-\exp \{[\mu(\beta)-\mu(\alpha)] /(\text { R.T })\}+\exp \{[\mu(\alpha)-\mu(\alpha)] /(\text { R.T })\}\}= \\
& \text { R.T.III.c }{ }_{0} /(\gamma . \delta) . \exp [-Æ /(R . T)] \text {. } \\
& \{1-\exp \{[\mu(\beta)-\mu(\alpha)] /(\text { R.T })\}\}= \\
& \text { R.T.III.c } /(\gamma . \delta) . \exp [-Æ /(R . T)] \text {. } \\
& \left\{1-\exp \left[\Delta_{\mathrm{r}} \cdot \mu /(\mathrm{R} . \mathrm{T})\right]\right\}= \\
& \text { R.T.III.c }{ }_{\mathrm{r}}^{\mathrm{r}} /(\gamma \cdot \delta) \cdot \exp [-Æ /(\text { R.T })] .\{1-\exp [-\mathrm{A} /(\mathrm{R} . \mathrm{T})]\}
\end{aligned}
$$

where $\Delta_{r} \mu=\mu(\beta)-\mu(\alpha)$ : total chemical potential change of the isothermal process, $\alpha \rightarrow \beta, A=\Delta_{\mathrm{r}} \mu$ : affinity of the process. Since $\Delta_{r} \mu<0$ in any spontaneous isothermal, isobaric process, the affinity, $\mathrm{A}$, of the process is always positive and finite.

Derived from irreversible thermodynamics and dimensional correctness verified (Appendix II), Eq. G, a unified global kinetic equation for isothermal materials processes (UPKE), states that the rate of any matter transformation/transport is, in general, nonlinearly related both to the activation energy and the affinity of the process. Accordingly, if a process is at (thermodynamic) equilibrium, i.e., $\mathrm{A}=0$, its rate, $\varrho$, must also be zero. On the other hand, a 'negligible' rate, @, of any highlyactivated $(Æ>>R T)$ process at temperature does not necessarily indicate an establishment of equilibrium of the process if the affinity, A, is not insignificant. Kinetic perceptions derived from the proposed thermodynamic modeling are thus consistent with those evolved from the classical kinetic theories of mass action and matter transport $[2,3,7,8]$.

\section{SIGNIFICANCE OF RESONANT WAVE-FIELDS ON ISOTHERMAL CERAMIC PROCESS KINETICS}

According to classical thermodynamics, the total chemical potential of a single-component ceramic at a given temperature, $\mathrm{T}$, and pressure, $\pi$, in a field-free (zero-field) environment is $[1,6]$ : 
$\mu^{\circ}=\mu^{\mathrm{T}, \pi}+\mu^{2}$

where $\mu^{\circ}, \mu^{\mathrm{T}, \pi}$, and $\mu^{2}$ are the total chemical potential, the bulk chemical potential, and the surface chemical potential of the dielectric ceramic body under any field-free, constant temperature, and constant pressure condition, respectively.

Resonant wave-fields and their effects on total chemical potential: effective field-energy used in ceramic processing is frequently cyclic, coherent, and resonant electromagnetic or acoustic in nature. As long as the frequencies of these incident waves, e.g., electromagnetic microwaves or acoustic ultrasonic waves, are significantly lower than the thermally-induced molecular/ionic vibrational frequency of the irradiated system, the local instantaneous equilibrium hypothesis allows all related intensive thermodynamic properties to be instantaneously defined at any meso-volume element (cell) within the overall nonequilibrium system [1, $4,5]$. In scenarios of any linear, isotropic, homogeneous ceramic dielectric body exposed to a uniform, coherent, and resonant wave-field, the time-averaged, molar work done by the resonant wave-field is expected to become as the fieldinduced chemical potential of the body, $\mu^{\mathrm{F}}$, as $[1,6]$ :

$\mu^{\mathrm{F}}=\mu^{\mathrm{MWF}}=<\mathrm{w}^{\mathrm{P}}>+<\mathrm{w}^{\mathrm{M}}>$

$=(\Omega / 4) \cdot\left[\varepsilon_{\mathrm{o}} \cdot\left(\varepsilon_{\mathrm{r}}^{\max }-1\right) \cdot \mathrm{E}_{\mathrm{o}}{ }^{2}\right]+(\Omega / 4) \cdot\left[\mu_{\mathrm{o}} \cdot\left(\mu_{\mathrm{r}}^{\max }-1\right) \cdot \mathrm{H}_{\mathrm{o}}{ }^{2}\right]$

for any exposure of the ceramic body to a resonant microwave field (MWF); or

$\mu^{\mathrm{F}}=\mu^{\mathrm{USF}}=<\mathrm{W}^{\mathrm{U}}>=2 \pi^{2} \cdot \Omega \cdot d \cdot(v \cdot \dot{\mathrm{S}})^{2}$

for any exposure of the ceramic body to a resonant ultrasonic wave-field (USF). In Eqs. Ia and Ib, $\left\langle\mathrm{w}^{\mathrm{P}}\right\rangle=(\Omega / 4)\left[\varepsilon_{0}\left(\varepsilon_{\mathrm{r}}^{\max }-1\right) \mathrm{E}_{\mathrm{o}}^{2}\right]$, $\left\langle\mathrm{w}^{\mathrm{M}}\right\rangle=(\Omega / 4)\left[\mu_{\mathrm{o}}\left(\mu_{\mathrm{r}}^{\max }-1\right) \mathrm{H}_{\mathrm{o}}{ }^{2}\right]$, and $\left\langle\mathrm{w}^{\mathrm{U}}\right\rangle=2 \pi^{2} \Omega \mathrm{d}\left(v_{\mathrm{s}}\right)^{2}$ are the time-averaged, resonant molar dielectric, magnetic, and ultrasonic (acoustic) work done on the body, respectively. Also, $\mathrm{E}_{\mathrm{o}}, \mathrm{H}_{\mathrm{o}}$ : amplitudes of the electric and magnetizing fields of the applied resonant microwave irradiation, respectively, $\varepsilon_{\mathrm{r}}^{\max }, \mu_{\mathrm{r}}^{\max }$, š: maximized relative permittivity, maximized relative permeability, and augmented cyclic molecular displacement under the resonant electric, magnetizing, and ultrasonic fields, respectively, $v$ : frequency of the applied resonant ultrasonic waves, $\Omega$, d: molar volume and mass density of the ceramic, respectively, and $\varepsilon_{o}, \mu_{o}$ : permittivity and permeability of vacuum, respectively.

In resonance physics of matter, the maximized relative permittivity, $\varepsilon_{\mathrm{r}}^{\max }$, and maximized relative permeability, $\mu_{\mathrm{r}}^{\max }$, in a resonant microwave field, as well as the augmented cyclic

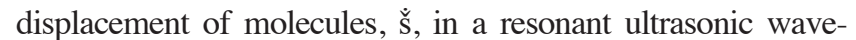
field, could be orders of magnitude higher than their nonresonant counterparts. It is thus anticipated that under a resonant microwave or ultrasonic wave-field, the field-created chemical potential, $\mu^{\mathrm{F}}$, could be comparable to typical solid-state molar bond-energy [1, 6, 9]. Consequently, if any one-component ceramic body is exposed to a uniform, resonant wave-field at a given temperature and pressure, the total chemical potential of the body, $\mu^{\mathrm{WF}}$, may be augmented and expressed as:
$\mu^{\mathrm{WF}}=\mu^{\circ}+\mu^{\mathrm{F}}=\mu^{\mathrm{T}, \pi}+\mu^{2}+\mu^{\mathrm{F}}$

Since $\mu^{\mathrm{F}}$ is a significant positive quantity, $\mu^{\mathrm{WF}}$, the total chemical potential of the ceramic body under a uniform resonant wave-field, as per Eq. J, may be considerably greater than the total chemical potential of the same ceramic under its field-free condition, $\mu^{\circ}[1,6]$. Consequently, a resonant wave-field could significantly promote the total chemical potential of a ceramic even at a condition of constant temperature, pressure, and composition as illustrated in Fig. 2: $\mu^{\circ}(\alpha)$ is promoted to $\mu^{\mathrm{WF}}(\alpha)$ in the initial-state $\alpha$ of the ceramic; $\mu^{\circ}(\beta)$ is elevated to $\mu^{\mathrm{WF}}(\beta)$ in the final-state $\beta$ of the ceramic. Both potential augmentations occur due to the applied resonant wave-field, WF.

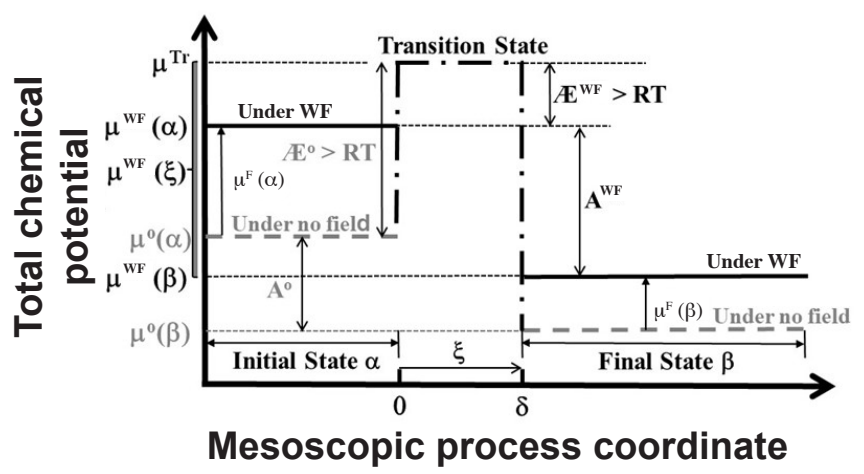

Figure 2: A schematic showing the total chemical potential variations along the mesoscopic paths of the isothermal ceramic processes under field-free and WF conditions. These two matter processes, both converting a ceramic from its initial-state $\alpha$ into its final-state $\beta$, are envisioned as individual diffusion processes, transferring transition complexes along a mesoscopic process coordinate, $\xi$, over the activation energy barriers, $\mathbb{E}^{\circ}$ and $Æ^{\mathrm{WF}}$, respectively, through the entire process-path-length, $\delta$, and subsequently into the final state. Note: both $\mathbb{E}^{\circ}$ and $\mathbb{E}^{\mathrm{WF}}>\mathrm{RT}$.

Enhancements of ceramic processes in resonant wavefields - a unified comprehension: promotion of total chemical potential of a ceramic system in a resonant wave-field may not only augment the isothermal thermodynamics (instability) of the system but may also enhance the isothermal kinetics of any macro-process occurring within the affected nonequilibrium ceramic body. Two measurable and controllable process kinetic factors (variables) accordingly affected in a resonant wave-field are the activation energy, $Æ$, and the affinity, A. Referring to Fig. 2, field-modified activation energy is expressed as $\varlimsup^{\mathrm{WF}}=Æ^{\circ}-\mu^{\mathrm{F}}(\alpha)$. Since $\mu^{\mathrm{F}}(\alpha)>0, Æ^{\mathrm{WF}}$ is always lower than its field-free counterpart, $\mathbb{E}^{\circ}$. Alternatively, the field-augmented affinity is expressed as $\mathrm{A}^{\mathrm{WF}}=\mathrm{A}^{\circ}-\left[\mu^{\mathrm{F}}(\beta)-\mu^{\mathrm{F}}(\alpha)\right]$. For any thermodynamically favorable process converting a ceramic from an initial-state $\alpha$ into its final-state $\beta$, the condition of $\mu^{\mathrm{F}}(\beta) \leq \mu^{\mathrm{F}}(\alpha)$ usually holds. Therefore, the affinity of a field-assisted process, $\mathrm{A}^{\mathrm{WF}}$, at a given temperature may be either greater or equal to that of its field-free counterpart process, $\mathrm{A}^{\circ}$. In general, the scenario of $\mathrm{A}^{\mathrm{WF}} \approx \mathrm{A}^{\circ}$ applies to non-reactive sintering and 
grain growth processes, whereas the scenario of $\mathrm{A}^{\mathrm{WF}}>\mathrm{A}^{\circ}$ most likely applies to physical phase transitions and chemical (compositional) transformations. Consequently, if one employs the knowledge of $Æ^{\mathrm{WF}}<\AA^{\circ}$ and $\mathrm{A}^{\mathrm{WF}} \geq \mathrm{A}^{\circ}$ in conjunction with the proposed UPKE, i.e., Eq. G, one may readily optimize and accelerate the global rate of any ceramic process at a given temperature by applying a proper resonant wave-field.

\section{CHARACTERIZING ISOTHERMAL CERAMIC PROCESS KINETICS UNDER FIELD-FREE AND RESONANT WAVE-FIELD CONDITIONS}

The global steady-state process rate of any isothermal ceramic process, $\varrho$, predicted by the proposed UPKE (Eq. $\mathrm{G})$, is nonlinearly related to two key process factors: the activation energy and the affinity. These two factors, on the other hand, are experimentally controllable and measurable. Hence, one could directly use the equation to characterize the kinetics of all types of isothermal ceramic processes. Yet to effectively apply the proposed UPKE to daily processing investigation, one may further categorize common ceramic processes into two main types, then characterize their kinetics individually using an appropriately simplified form of the UPKE as derived in Appendix III.

Type I - matter-transport-controlled processes (physical) structural transitions): most matter-transport-controlled processes in ceramics are operated by slow solid-state diffusion mechanisms, which are typically driven by a very low affinity $[6,10]$, i.e., $\mathrm{A} /(\mathrm{RT})<10^{-2}$. Examples are isothermal non-reactive sintering and related processes [11$15]$, and glass-crystallization $[16,17]$ under field-free and resonant wave-field conditions. Characterizing the kinetics of these processes may therefore use the approximated form of the UPKE or Eq. 7 derived in Appendix III:

$\mathrm{Q}^{\mathrm{T}}=$ III.c. $/(\gamma \cdot \delta) \cdot \exp [-Æ /(\mathrm{R} \cdot \mathrm{T})] \cdot \mathrm{A}=\mathrm{K}^{\mathrm{T}} \cdot \exp [-Æ /(\mathrm{R} \cdot \mathrm{T})] \cdot \mathrm{A}$

where $\mathrm{Q}^{\mathrm{T}}$ is the global steady-state rate of any isothermal matter-transport-controlled process in a ceramic, and $\mathrm{K}^{\mathrm{T}}=\mathrm{IIIC}_{\mathrm{o}} /(\gamma \delta)$ is a material constant for the transport process at a given temperature. Eq. Ga suggests that the process rates of these Type I ceramic processes are linearly related to their corresponding affinity in the spirit of Fick's mass diffusion law. The experimentally measurable process factors, $Æ$ and $\mathrm{A}$, both determine the kinetics of these processes (Fig. 3). Any process rate enhancement in a resonant wave-field in this scenario is, therefore, attributable to one of the following two reasons: i) a sole reduction in activation energy of the process induced by the resonant field [1] as experimentally demonstrated in microwave sintering of oxides [13-15], e.g., Æ of sintering was observed to reduce from $575 \mathrm{~kJ} / \mathrm{mol}$ (conventional heating) to $160 \mathrm{~kJ} / \mathrm{mol}$ (microwave heating) in high-purity alumina, also from $\sim 500 \mathrm{~kJ} / \mathrm{mol}$ (conventional heating) to $\sim 200 \mathrm{~kJ} / \mathrm{mol}$ (microwave heating) in yttria-stabilized zirconia, or ii) a field-induced activation energy reduction accompanied by field-augmented affinity during the process [1] as observed in microwave-assisted lithium disilicate glass-crystallization - the glass, in microwave heating, completely crystallized within 2 min at $600{ }^{\circ} \mathrm{C}$, whereas in conventional heating, full crystallization required a significantly higher temperature $\left(680^{\circ} \mathrm{C}\right)$ and considerably longer time $(100 \mathrm{~h})[16,17]$.

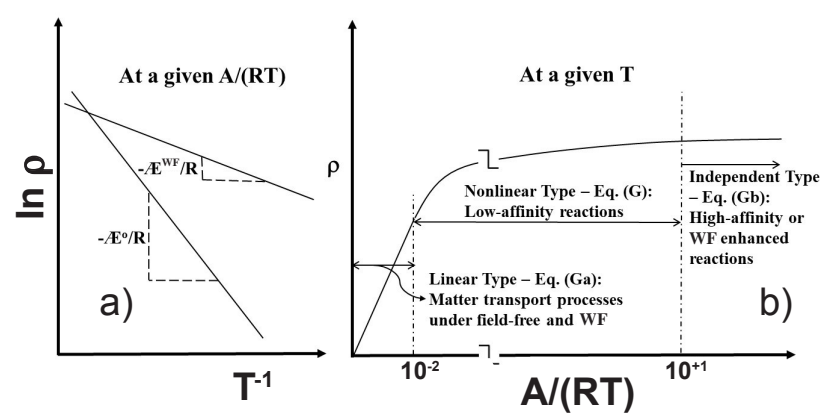

Figure 3: Not-to-scale schematics demonstrating key process factors which characterize and control isothermal kinetics of ceramic processes under field-free and resonant wave-field conditions: a) activation energy, $Æ$, for all types of processes, and b) affinity, A, particularly for relatively low-affinity processes with $\mathrm{A} /(\mathrm{RT})<10$.

Type II - reactions (chemical/compositional transformations): most isothermal solid-state (chemical) reactions in ceramic processing, including those occurring under resonant wave-fields, possess a high affinity $[6,10]$, i.e., $\mathrm{A} /(\mathrm{RT})>10$. The kinetics of this type of processes may thus be described by the degenerate form of the proposed UPKE or Eq. 8 derived in Appendix III:

$\mathrm{Q}^{\mathrm{R}}=$ R.T.III.c. $/(\gamma \cdot \delta) \cdot \exp [-Æ /(\mathrm{R} . \mathrm{T})]=\mathrm{K}^{\mathrm{R}} \cdot \exp [-Æ /(\mathrm{R} . \mathrm{T})]$

where $Q^{R}$ is the global steady-state process rate of an isothermal, high-affinity reaction occurring in a ceramic, and $K^{R}=R T I I I c_{0} /(\gamma . \delta)$ is a process constant for the reaction at a given temperature. Eq. Gb predicts that the rate of any (highaffinity) Type II ceramic process is generally independent of its affinity, as demonstrated in Fig. 3b. Consequently, its rate is solely governed by a negative exponential function of its activation energy in the spirit of Arrhenius's reaction rate law as illustrated in Fig. 3a. As a matter of fact, reducing activation energy using an appropriate resonant wave-field is a widely recognized procedure in achieving isothermal kinetics enhancements of solid-state reactions in ceramic processing. Using microwaves, for example, the isothermal kinetics of $\mathrm{CuO}$ reduction reaction was reported to be greatly enhanced solely due to a field-induced decrease in the activation energy of the process from $328 \mathrm{~kJ} / \mathrm{mol}$ (conventional heating) to $117 \mathrm{~kJ} / \mathrm{mol}$ (microwave heating) $[18,19]$. On the other hand, enhanced rates of the solid-state reaction in the $\mathrm{Fe}_{2} \mathrm{O}_{3}-\mathrm{Li}_{2} \mathrm{CO}_{3}$ system conducted under ultrasonic waves between 400 and $500{ }^{\circ} \mathrm{C}$ were also demonstrated to result merely from a field-induced activation energy reduction from $120 \mathrm{~kJ} / \mathrm{mol}$ (without ultrasonic waves) to $65 \mathrm{~kJ} / \mathrm{mol}$ (in an ultrasonic field) [20]. 


\section{CONCLUDING REMARKS}

Based on mesoscopic irreversible thermodynamics, a unified comprehension of isothermal materials process kinetics was first developed, followed by the derivation of a unified process kinetic equation (UPKE). The proposed UPKE affirms that the global rate of any isothermal materials process is, in general, a nonlinear function of both the activation energy and affinity of the process. Further, perceptions and rationale evolved from this UPKE are consistent with those developed from the classical kinetic theories of mass action and mass transport. Accordingly, one may use an aptly simplified form of the proposed UPKE to properly understand, characterize, and optimize the kinetics of most ceramic processes conducted under field-free and resonant wave-field conditions. For a low-affinity, matter-transport-controlled (Type I) process, the global isothermal process rate, as advised by the approximated form of the proposed UPKE, is simply linearly related to its affinity. Hence, the kinetics enhancement of a low-affinity, diffusion-controlled ceramic process conducted in a resonant wave-field is expected to be caused either by a reduction in activation energy, as widely accepted in microwave-sintering of oxides, or by activation energy reduction together with affinity augmentation, as revealed in microwave-assisted glasscrystallization. Conversely, global kinetics of any isothermal, high-affinity (Type II) process, as per the degenerate form of the UPKE, is primarily controlled by the activation energy of the process. Hence, process rate enhancement of any high-affinity, reaction-controlled ceramic process under a resonant wavefield is considered to result directly from reduced activation energy attributable to the field-induced promotion of the total chemical potential of the initial-state matter during the process.

\section{ACKNOWLEDGMENT}

This research was self-motivated and self-sponsored by the author. It did not receive any grants from funding agencies in the public, commercial, or not-for-profit sectors.

\section{MAJOR SYMBOLS}

\author{
A: affinity \\ $\mathrm{A}^{\circ}$ : field-free affinity \\ $\mathrm{A}^{\mathrm{WF}}$ : resonant field-augmented affinity \\ Æ: activation energy \\ $\mathbb{E}^{\circ}$ : field-free activation energy \\ $\mathbb{E}^{\mathrm{WF}}$ : resonant field-modified activation energy \\ $\mathrm{c}_{\mathrm{o}}$ : standard-state concentration \\ $\mathrm{c}(\xi)$ : transition complex concentration along $\xi$ \\ $\mathrm{T}$ : thermodynamic temperature \\ $\alpha$ : initial or reactant state \\ $\beta$ : final or product state \\ $\gamma$ : activity coefficient \\ $\delta$ : length of a meso-process-path \\ $\lambda$ : phenomenological kinetic coefficient \\ $\mu$ : total chemical potential \\ $\mu^{\circ}$ : total chemical potential under a field-free condition
}

$\mu^{\mathrm{F}}$ : resonant field-induced chemical potential

$\mu^{\mathrm{WF}}$ : total chemical potential in a resonant wave-field

$\xi$ : isothermal, mesoscopic/internal process coordinate $\pi$ : pressure

@: global, isothermal, steady-state process rate

$\varrho(\xi)$ : local or mesoscopic, isothermal process rate

$\sigma(\xi)$ : entropy production rate of a meso-volume cell

III: molar mobility

\section{APPENDICES}

I) Evaluating total chemical potential along a mesoscopic process coordinate - a thermodynamic analysis: originally proposed in [6], this analysis is now revised and completed as follows: at constant pressure $(\pi)$ conditions, change in total chemical potential of a (pure) solid component (substance) with respect to temperature is:

$(\partial \mu / \partial \mathrm{T})_{\pi}=-\mathrm{s}$

where $\mu$ : total chemical potential, s: molar entropy, and T: thermodynamic temperature. Now, considering an isobaric process: converting the component from its initial-state $\alpha$ into a final-state $\beta$ along any mesoscopic process coordinate (path), $\xi$, one may express the total chemical potential change during the process as per Eq. 1:

$\{\partial[\mu(\xi)-\mu(\alpha)] / \partial \mathrm{T}\}_{\pi}=-[\mathrm{s}(\xi)-\mathrm{s}(\alpha)]$

where $\mu(\xi), \mu(\alpha)$ : total chemical potentials of the transition complex (the 'transient-state' component) along the process path, $\xi$, and the component at its initial state, $\alpha$, respectively; $\mathrm{s}(\xi), \mathrm{s}(\alpha)$ : molar entropies of the transition complex and the initial-state component, respectively. On the other hand, given that the chemical potential of any component in an isothermal and isobaric environment is:

$\mu=\mu_{\mathrm{o}}+$ R.T.Ina

where $\mathrm{a}$ is the activity and $\mu_{\mathrm{o}}$ is the standard-state chemical potential. Applying Eq. 3 to Eq. $2, \partial[\mu(\xi)-\mu(\alpha)] / \partial$ T may now be written as:

$\partial[\mu(\xi)-\mu(\alpha)] / \partial \mathrm{T}=\partial\{\mathrm{R} \cdot \mathrm{T} \cdot \ln [\mathrm{a}(\xi) / \mathrm{a}(\alpha)]\} / \partial \mathrm{T}=-[\mathrm{s}(\xi)-\mathrm{s}(\alpha)](4 \mathrm{a})$

or simply:

$\mathrm{R} \cdot \ln \mathrm{a}(\xi)=-[\mathrm{s}(\xi)-\mathrm{s}(\alpha)]=-\Delta \mathrm{s}$

where $\mathrm{a}(\xi), \mathrm{a}(\alpha) \equiv 1$ : activities of the transition complex along $\xi$ and the pure $\alpha$-state component, respectively. Furthermore, referring to Fig. 1, the total chemical potential change of the isothermal, isobaric process, $\alpha \rightarrow \beta$, along the mesoscopic coordinate, $\xi$, as per Gibbs's formalism, is:

$\mu(\xi)-\mu(\alpha)=[h(\xi)-h(\alpha)]-T .[s(\xi)-s(\alpha)]$ 
where $h(\xi), h(\alpha)$ : molar enthalpies of the transition complex and the initial-state $\alpha$ component, respectively. Given that activation energy/enthalpy $Æ(\xi) \equiv[\mathrm{h}(\xi)-\mathrm{h}(\alpha)]$ and $Æ(\xi)=Æ=$ constant $>$ RT in the domain of $0<\xi<\delta$ as illustrated in Fig. 1, one, in conjunction with Eq. 4b, may rewrite Eq. 5 as:

$\mu(\xi)-\mu(\alpha)=Æ-T .[s(\xi)-s(\alpha)]=Æ+R \cdot T \cdot \operatorname{lna}(\xi)$

Since $\mathrm{a}(\xi) \equiv \gamma . \mathrm{c}(\xi) / \mathrm{c}_{0}$, one may thus express Eq. 6 as:

$\mu(\xi)-\mu(\alpha)=Æ+R \cdot T \cdot \ln \left[\gamma . c(\xi) / \mathrm{c}_{\mathrm{o}}\right]$

Via a subsequent term-rearrangement, the total chemical potential of the transition complex along the mesoscopic process coordinate, $\xi$, may be stated as:

$\mu(\xi)=\mu(\alpha)+R \cdot T \cdot \ln \left[\gamma \cdot c(\xi) / c_{0}\right]+Æ$

where $c(\xi)$ : concentration of the transition complex along $\xi ; \mathrm{c}_{\mathrm{o}} \equiv 1 \mathrm{M}=1 \mathrm{kmol} / \mathrm{m}^{3}$ at a given temperature under the standard-state pressure (1 atm); and $\gamma$ : activity coefficient.

II) Verifying dimensional correctness of the proposed UPKE (Eq. G): 1) Dimensions and units: i) SI units of relevant primary dimensions: length $(\mathrm{L})$ : $\mathrm{m}$; time $(\mathrm{t})$ : $\mathrm{s}$; amount of matter $(\mathrm{N})$ : mol; temperature (T): K; ii) SI units of relevant derived (nonprimary) dimensions: universal gas constant (R): J.mol ${ }^{-1} \cdot \mathrm{K}^{-1}$; molar mobility (III): m.mol. $\mathrm{s}^{-1} \cdot \mathrm{N}^{-1}$; concentration (c): mol.m ${ }^{-3}$; activation energy (Æ): J.mol ${ }^{-1}$; affinity (A): $\mathrm{J}^{\mathrm{mol}}{ }^{-1}$. 2) Dimensional correctness verification of the rate, $\varrho$, in the UPKE (Eq. G). Given that the exponential functions, $\exp [-Æ /(\mathrm{RT})]$ and $\exp [-\mathrm{A} /(\mathrm{RT})]$, in the Eq. $\mathrm{G}$ are dimensionless, the dimension of the rate, $\mathrm{Q}$, symbolized as [@], is thus solely determined by the dimension of the factor, $\left(\right.$ RTIII $\left.c_{\alpha}\right) /(\gamma \delta)$, symbolized as [(RTull, $\left.) /(\gamma \delta)\right]$, where $\gamma$ is a dimensionless constant: [@] $=\left[\left(\mathrm{Jmol}^{-1} \mathrm{~K}^{-1}\right) \mathrm{K} \cdot\left(\mathrm{mmols}^{-1} \mathrm{~N}^{-1}\right) \cdot\left(\mathrm{mol} \cdot \mathrm{m}^{-3}\right) \mathrm{m}^{-1}\right]$; considering $\mathrm{J}=\mathrm{N} \cdot \mathrm{m},[\mathrm{Q}]=\left[\mathrm{mol} \cdot \mathrm{m}^{-2} \cdot \mathrm{s}^{-1}\right]$. Consequently, the rate, $\mathrm{Q}$, in the UPKE has a dimension measuring amount of matter transformed or transported per unit area in a unit of time.

III) Deriving simplified forms of the UPKE for daily-encountered ceramic process characterization: in order to effectively apply the UPKE, i.e., Eq. G, proposed in this paper to characterize ceramic process kinetics, one may first categorize daily-encountered ceramic processes into two main types, then analyze their individual kinetics using an aptly simplified form of the equation as suggested below. 1) Approximated UPKE for Type I low-affinity process kinetics: for any low-affinity ceramic process with $\mathrm{A} /(\mathrm{RT})<10^{-2}$, the process rate, $Q$, predicted by the UPKE can be effectively approximated as:

$\mathrm{Q}=$ R.T.III.c $/(\gamma \cdot \delta) \cdot \exp [-Æ /($ R.T $)] \cdot\{1-\exp [-\mathrm{A} /(\mathrm{R} . \mathrm{T})]\}$

$\approx$ R.T.III.c $/(\gamma . \delta) \cdot \exp [-Æ /(R . T)] .\{1-[1-\mathrm{A} /(\mathrm{R} . \mathrm{T})]\}$

$=$ III.c. $/(\gamma \cdot \delta) \cdot \exp [-Æ /(R \cdot T)] \cdot A$
Therefore, Eq. 7 suggests that the rate of a low-affinity ceramic process such as sintering or glass-crystallization is linearly related to its affinity in the spirit of Fick's mass diffusion law. 2) Degenerate UPKE for Type II high-affinity process kinetics: in scenarios of high-affinity ceramic processes with $\mathrm{A} /(\mathrm{RT})>10$, the UPKE 'degenerates'. Hence mathematically, it may be simplified to:

$\mathrm{Q}=$ R.T.III.c. $/(\gamma \cdot \delta) \cdot \exp [-Æ /($ R.T $)] \cdot\{1-\exp [-\mathrm{A} /(\mathrm{R} \cdot \mathrm{T})]\}$

$\approx$ R.T.III.c $/(\gamma \cdot \delta) \cdot \exp [-Æ /(R . T)] .(1-0)$

$=$ R.T.III.c. $/(\gamma \cdot \delta) \cdot \exp [-Æ /(R \cdot T)]$

As a result, Eq. 8 predicts that the rate of a high-affinity ceramic process, e.g., a chemical reaction, is virtually independent of its affinity. Instead, its kinetics is solely governed by its activation energy in the spirit of Arrhenius's reaction rate law.

\section{REFERENCES}

[1] B. Wong, Ceram. Trans. 266 (2019) 181.

[2] I. Prigogine, Introduction to thermodynamics of irreversible processes, Charles C. Thomas Publ., Springfield (1955).

[3] D. Kondepudi, I. Prigogine, Modern thermodynamics, $2^{\text {nd }}$ ed., John Wiley Sons, West Sussex (2015).

[4] S.R. de Groot, P. Mazur, Non-equilibrium thermodynamics, Dover Publ., New York (1984).

[5] A. Katchalsky, P.F. Curran, Nonequilibrium thermodynamics in biophysics, Harvard Un. Press, Cambridge (1965).

[6] B. Wong, Proc. MS\&T19, Portland (2019) 174.

[7] N. Tu, J.W. Mayer, L.C. Feldman, Electronic thin film science, Macmillan Publ., New York (1992).

[8] D.S. Wilkinson, Mass transport in solids and fluids, Cambridge Un. Press, Cambridge (2000).

[9] B. Wong, Proc. $2^{\text {nd }}$ Global Congr. Microw. Energy Appl., R.L. Schulz, D.C. Folz (Eds.), 48HrBooks, USA (2013) 204. [10] M.F. Ashby, D.R.H. Jones, Engineering materials 2, $2^{\text {nd }}$ ed., Butterworth-Heinemann, Oxford (1998).

[11] G.C. Kuczynski, N.A. Hooton, C.F. Gibbon, Sintering and related phenomena, Gordon Breach Sci. Publ., New York (1967).

[12] S. Somiya, Y. Moriyoshi (Eds.), "Sintering, key papers", Elsevier Sci. Publ., Essex (1990).

[13] M.A. Janney, H.D. Kimrey, Proc. Microw. Proces. Mater. II, 189, MRS, USA (1991) 215.

[14] K.H. Brosnan, G.L. Messing, D.K. Agrawal, J. Am. Ceram. Soc. 86, 8 (2003) 1307.

[15] R.R. Thridandapani, "The effect of microwave energy on sintering", PhD Thesis, Virginia Polytech. Inst. State Un., Blacksburg (2011).

[16] M.M. Mahmoud, "Crystallization of lithium disilicate glass using variable frequency microwave processing", $\mathrm{PhD}$ Diss., Virginia Polytech. Inst. State Un., Blacksburg (2007). [17] M.M. Mahmoud, D.C. Folz, C.T.A. Suchicital, D.E. Clark, J. Am. Ceram. Soc. 95, 2 (2012) 579. 
[18] M. Sato, J. Fukushima, S. Takayama, K. Nagata, K. Hara, M. Hotta, Y. Kitamura, M. Hayashi, N. Shinohara, T. Mitani, K. Kashimura, MS\&T11 Conf., Columbus (2011).

[19] M. Sato, J. Fukushima, K. Kashimura, T. Mitani, D.
Agrawal, Proc. $2^{\text {nd }}$ Global Congr. Microw. Energy Appl., R.L. Schulz, D.C. Folz (Eds.), 48HrBooks, USA (2013) 112. [20] A.E. Baranchikov, V.K. Ivanov, N.N. Oleinikov, Y.D. Tretyakov, Russian J. Inorg. Chem. 48, 1 (2003) 35.

(Rec. 04/08/2020, Rev. 10/09/2020, Ac. 15/09/2020) 